Article

\title{
Suppression of SHROOM1 Improves In Vitro and In Vivo Gene Integration by Promoting Homology-Directed Repair
}

\author{
Zhihua Zhao ${ }^{1}$, , Hanshuo Zhang ${ }^{2}$, Tuanlin Xiong ${ }^{3}$, Junyi Wang ${ }^{2}$, Di Yang ${ }^{2}$, Dan Zhu ${ }^{2}$, \\ Juan $\mathrm{Li}^{2}$, Ye Yang ${ }^{2}$, Changhong Sun ${ }^{2}$, Yuting Zhao ${ }^{1}$ and Jianzhong Jeff $\mathrm{Xi}^{1,2}$,* \\ 1 State Key Laboratory of Biomembrane and Membrane Biotechnology, Institute of Molecular Medicine, \\ Peking University, Beijing 100871, China; zhaozhihua1831@126.com (Z.Z.); ytzhao2014@163.com (Y.Z.) \\ 2 Department of Biomedical Engineering, College of Engineering, Peking University, Beijing 100871, China; \\ shzzhshuo@sina.com (H.Z.); wangjunyi1994@pku.edu.cn (J.W.); coeyangdi@pku.edu.cn (D.Y.); \\ zhudanzd1991@126.com (D.Z.); jinyujin_0@163.com (J.L.); yangye8686@163.com (Y.Y.); \\ sunchanghong1983@163.com (C.S.) \\ 3 College of Life Science, Peking University, Beijing 100871, China; xiongtuanlin@mail.tsinghua.edu.cn \\ * Correspondence: jzxi@pku.edu.cn; Tel.: +86-10-6276-0698
}

Received: 25 June 2020; Accepted: 11 August 2020; Published: 13 August 2020

\begin{abstract}
Homologous recombination (HR) is often used to achieve targeted gene integration because of its higher precision and operability compared with microhomology-mediated end-joining (MMEJ) or non-homologous end-joining (NHEJ). It appears to be inefficient for gene integration in animal cells and embryos due to occurring only during cell division. Here we developed genome-wide high-throughput screening and a subsequently paired crRNA library screening to search for genes suppressing homology-directed repair (HDR). We found that, in the reporter system, HDR cells with knockdown of SHROOM1 were enriched as much as 4.7-fold than those with control. Down regulating SHROOM1 significantly promoted gene integration in human and mouse cells after cleavage by clustered regularly interspaced short palindromic repeats (CRISPR)/CRISPR-associated protein-9 nuclease (Cas9), regardless of the donor types. The knock-in efficiency of mouse embryos could also be doubled by the application of SHROOM1 siRNA during micro-injection. The increased HDR efficiency of SHROOM1 deletion in HEK293T cells could be counteracted by YU238259, an HDR inhibitor, but not by an NHEJ inhibitor. These results indicated that SHROOM1 was an HDR-suppressed gene and that the SHROOM1 knockdown strategy may be useful for a variety of applications, including gene editing to generate cell lines and animal models for studying gene function and human diseases.
\end{abstract}

Keywords: homologous recombination; SHROOM1; knock-in; gene editing; CRISPR/Cas9

\section{Introduction}

Targeted gene integration is usually achieved by a method based on homologous recombination (HR) [1-3]. Highly efficient integration requires a designed nuclease to generate the specific DNA double-strand break (DSB) and a repair template that harbors left and right homology arms (HAs) (100-3000 bp). Custom-designed nucleases include zinc-finger nucleases (ZFN) [4,5], transcription activator-like effector nucleases (TALEN) [2,6], the clustered regularly interspaced short palindromic repeats (CRISPR)/CRISPR-associated protein-9 nuclease (Cas9) system [3,7,8], and the CRISPR-Cpf1 (Cas12a) system $[9,10]$. Once a DSB is created, externally supplied DNA fragments can be introduced around the cleavage site to participate in the HR repair. Other cell repair pathways like non-homologous end-joining (NHEJ) or microhomology-mediated end-joining (MMEJ) are also used for targeted gene integration. The former seems to elevate the efficiency obviously, but it is unable to form a precise 
replacement and sometimes results in unforeseeable indels, making it difficult to generate endogenous and exogenous in-frame fusion genes for chimeric protein production [11,12]. The latter can manage accurate integration easily, but has low efficiency $[13,14]$.

HR-mediated gene integration is widely used, but is still inefficient in cells and embryos because HR only happens during the late $S / G 2$ phase $[15,16]$. As a result, how to enhance the efficiency has become a focus of current research. DNA donor engineering techniques such as chemical modification [17-19] or double-cut site-contained [20,21] have achieved greater efficiency. Approaches that push the cell to choose the HR pathway, like the application of the NHEJ inhibitor [22,23] or cell cycle key factor [21,24], increased integration to an extent. Similarly, higher expression of the HR-essential protein [25-27], or fusing it with a custom-designed nuclease $[25,28]$, could improve the efficiency.

There have rarely been reports about HR-suppressed genes and their usage in targeted gene integration, therefore, in this study, we conducted for the first time a genome-wide, high-throughput screening of genes regulating HR, and subsequently carried out paired crRNA library screening to look for HR-suppressed genes. We obtained SHROOM1 and further tested whether its knockdown could increase HDR efficiency in mammalian cells and embryos. We found that down-regulating SHROOM1 with siRNA could elevate HDR efficiency up to 3-fold in cultured cells, and significantly improve knock-in efficiency in mouse embryos.

\section{Results}

\subsection{Genome-Wide Screening is Conducted to Search for Genes that Modulate HDR}

To identify the factors modulating HDR efficiency at the DSB site, we modified a previously described fluorescence-based system that measures I-SceI-induced HDR events to carry out a genome-wide screening [29]. This system consists of a Tet-On [30] expression of I-SceI endonuclease and a reporter that contained one inactivated green fluorescent protein (GFP) copy linked with an internal GFP fragment (Figure 1a and Figure S1a (Supplementary Materials)). When doxycycline (Dox) was added, the reverse tetracycline transcriptional activator (rtTA) bound to the tet-responsive element (TRE) and changed the conformation to initiate the expression of I-SceI. Then, the inactivated GFP with $18 \mathrm{bp}$ I-SceI insertion was incised and conducted an HDR event, utilizing the distal GFP fragment to restore functional GFP expression (Figure 1a and Figure S1b (Supplementary Materials)).

Using human osteosarcoma cell, U2OS, stably expressing the above system (iDR-GFP U2OS), we examined the levels of HDR modulation induced by individually transfecting the siRNAs of 21,257 genes by a high-content analysis instrument and calculation of fold change (FC) (Figure 1b). After treating with siRNA or NC and inducing by Dox, in each field, cells with the HDR activity which exhibited green fluorescence would be counted as N. (GFP ${ }^{+}$cells). While total cells were stained with 4',6-diamidino-2-phenylindole (DAPI) and counted as N. (total cells) (Figure S1c (Supplementary Materials)). Six adjacent fields from a same sample were collected, and final HDR-ratio would be calculated by the ratio of N. (GFP+ cells) to N. (total cells). We used relative FC of HDR-ratio to define the ability of a gene affecting HDR (Figure 1b). BRCA2 [31,32] and RAD51 [32,33], critical factors potentiating recombinational DNA repair, were used as positive controls, and their siRNAs notably decreased the HDR fluorescence and resulted in FC $=0.48$ or FC $=0.41$, respectively, compared with NC siRNA (Figure 1c). In addition, the fluorescence brightness of two randomly selected genes (SSTR3 and SLC36A3) matched the reading data well, which represented high accuracy of our screening (Figure 1c). Our screening results showed that 367 siRNAs led to $F C<0.5$, including well-known components of DNA resection and repair (CtIP [34,35] and BRCA1 [31,35]), suggesting that these genes may maintain or promote HDR (Figure 1d, Table S4 (Supplementary Materials)). On the other hand, there were 1347 genes where siRNAs resulted in FC $>1.5$. These genes may play an important role in suppressing HDR progress in cells. 
a

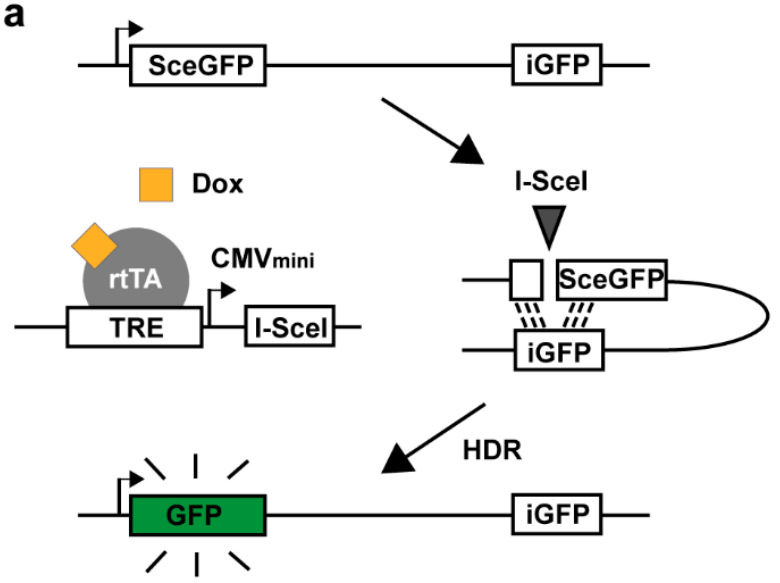

c

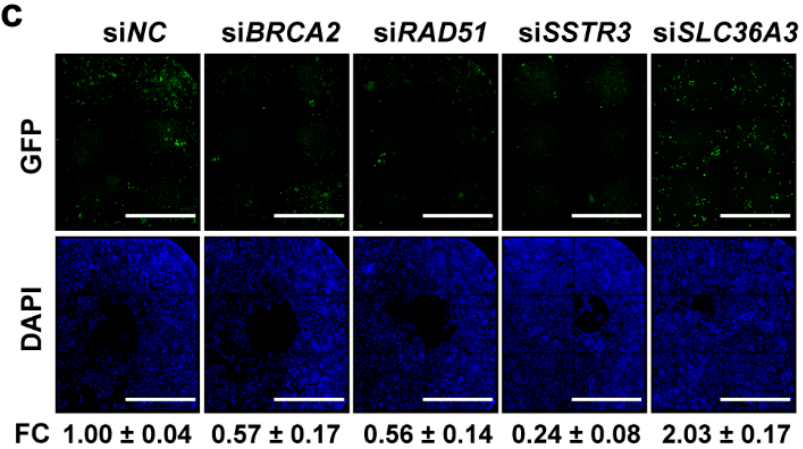

b

iDR-GFP U2OS

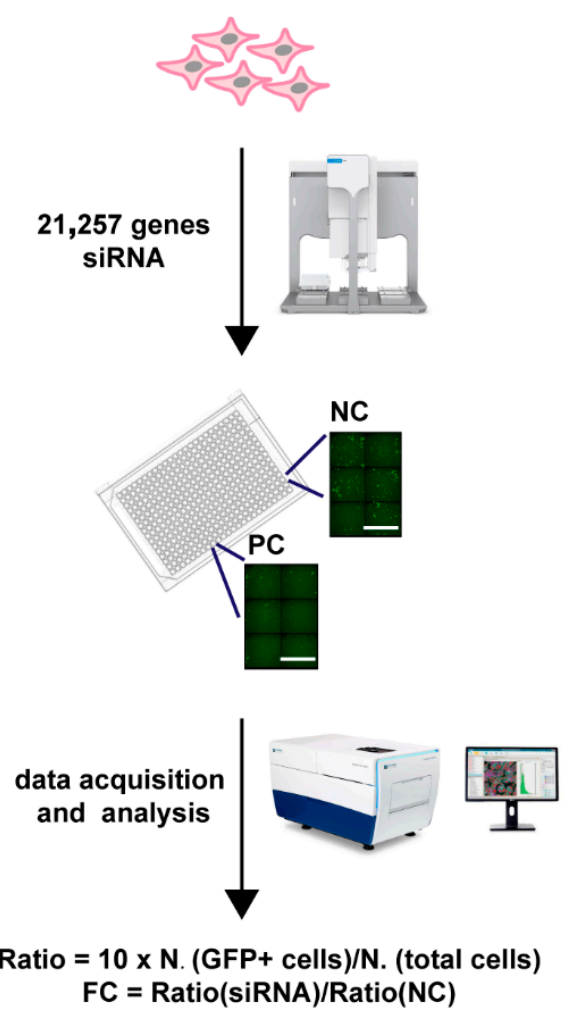

d

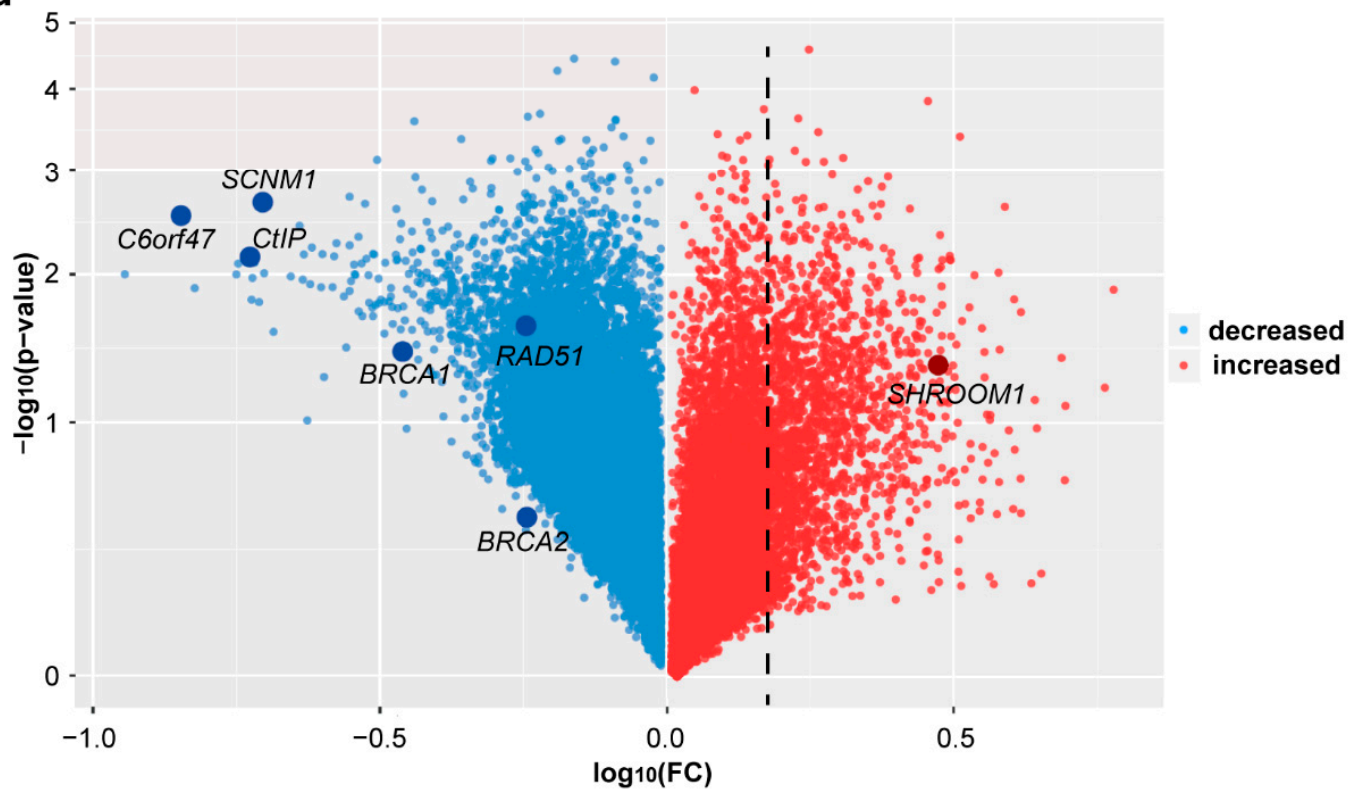

Figure 1. Genome-wide screening of HDR (homology directed repair)-modulated genes. (a) Schematic overview of the HDR progress in induced DR-GFP U2OS cells. Dox, doxycycline. (b) A flow diagram of the genome-wide screening using a high-content instrument. PC, positive control, BRCA2 and $R A D 51$; NC, negative control. Scale bar, $1000 \mu \mathrm{m}$. (c) Fluorescence pictures captured by a high-content instrument. FC, fold change. Every picture was stitched together from individual fields of vision. Scale bar, $1000 \mu \mathrm{m}$. (d) Multiple HDR-increased and decreased genes in the genome-wide screening. Representative genes are highlighted in dark blue. FC (fold change) of genes on dashed line equals 1.5. Data were generated from $n=3$ independent experiments. The $p$-value was calculated using a two-sided Student's $t$-test. 


\subsection{Suppressing SHROOM1 Promotes HDR in BFP-293T Cells}

In order to confirm that primary genes were suppressing HDR, we combined a paired-crRNA library targeting the 1347 genes mentioned above and 37 controls (11,918 pairs, eight pairs targeting each primary gene transcript) with the dual-cut blue fluorescent protein (BFP) reporter [36] cells for HDR to carry out a further screening (Figure 2a). Each cell in the screening pool stably expressed a copy of Acidaminococcus sp. BV3L6 Cpf1 [10], as well as mono-paired crRNAs targeting the promoter or splicing site of a single gene, respectively [37] (Figure S2a (Supplementary Materials)). To ensure the reliability of the screening, we first validated the editing efficiency of the integrated Cpf1 and mono crRNA pairs in HEK293T cells (Figure S2b (Supplementary Materials)). Genotyping and sequencing results showed that, after 28 days, the single paired crRNA copy of UPF1 could guide Cpf1 to generate effective editing including single cutting and long fragment deletion (Figure S2c,d (Supplementary Materials)). This pool was then transfected with Streptococcus pyogenes Cas9 and sgRNA vectors targeting the BFP reporter gene. After cleavage, the dissociative fragment was inverted to act as a homologous donor for gene repair, causing HEK293T cells to turn from blue to green (Figure S2e (Supplementary Materials)). Editing outcomes in each cell were separated by fluorescence-activated cell sorting (FACS), and Illumina sequencing was used to determine the genes whose knockout leads to enrichment or depletion from each sorted population.

Under unperturbed conditions, this combination of reporter and CRISPR system yielded $\sim 9.5 \%$ HDR (BFP edited to GFP) (Figure S2e (Supplementary Materials)). To identify the genes involved in editing events, we used FACS to separate cells into unedited $\left(\mathrm{BFP}^{+}\right)$and $\mathrm{HDR}\left(\mathrm{GFP}^{+}\right)$populations (Figure 2a). We recovered the crRNA abundance in each population by Illumina sequencing and compared these distributions to the edited unsorted populations to reveal which targeted gene increased (crRNA was enriched from the HDR population, but deleted from the BFP population) HDR activities. Our screening uncovered many genes promoting HDR (after crRNA editing), such as SHROOM1 and SLC36A3 (Figure 2b, Table S5 (Supplementary Materials)). SHROOM1 belongs to SHROOM family and mainly involves in the assembly of microtubule arrays during cell elongation [38,39], SHROOM1 interacts with F-actin to ensure the development of neuroepithelial cells [40], and regulates gamma-tubulin distribution and microtubule architecture during epithelial cell shape change [41]. SLC36A3 is belonging to SLC36 family and responsible for amino acid and proton transport [42,43].

We designed specific siRNAs for the top eight genes whose crRNAs were enriched in the HDR population in order to validate the results of our screening. These siRNA showed efficient suppression to corresponding genes in HEK293T cells (Figure S3a (Supplementary Materials)). Each siRNA was co-transfected with SpCas9 and sgRNA into the dual-cut BFP reporter cells, and the relative HDR ratio was calculated compared to NC by FACS. Four siRNAs exhibited obviously increased HDR efficiency, especially SHROOM1 (Figure 2c and Figure S3b (Supplementary Materials)). An additional three siRNAs for each effective gene were used in dual-cut BFP reporter cells to exclude the interference of off-target genes that similarly improved the HDR ratio (Figure S3c (Supplementary Materials)).

In order to determine the HDR enhancement was actually induced by gene editing, we combined the paired crRNAs of four genes that increased in validated results with reporter cells to construct monoclonal cell lines, as well as AAVS1 as a control. Since the 20th day after construction of the cell line, the HDR ratio of each cell line with SpCas9 system incision was recorded every two days (Figure 2d). The time curve showed that the HDR ratio of all cell lines experienced a 1.5-fold elevation compared to AAVS1 from day 24 (Figure 2d). Genome sequencing analysis indicated that the targeting sites of crRNA pairs were edited efficiently (Figure S3d (Supplementary Materials)). Apparent deletions around the two targets occurred in the SHROOM1 paired crRNA expressed cell line (Figure S3e (Supplementary Materials)). We mixed all 30-day monoclonal cell lines in a 1:1 ratio to simulate a mini-screening to find the one most enriched in HDR activity. As a result, the SHROOM1 crRNA was enriched 4.7-fold more than AAVS1 and therefore SHROOM1 was selected as a potent suppressor of HDR in these reporter cells (Figure 2e). 
a
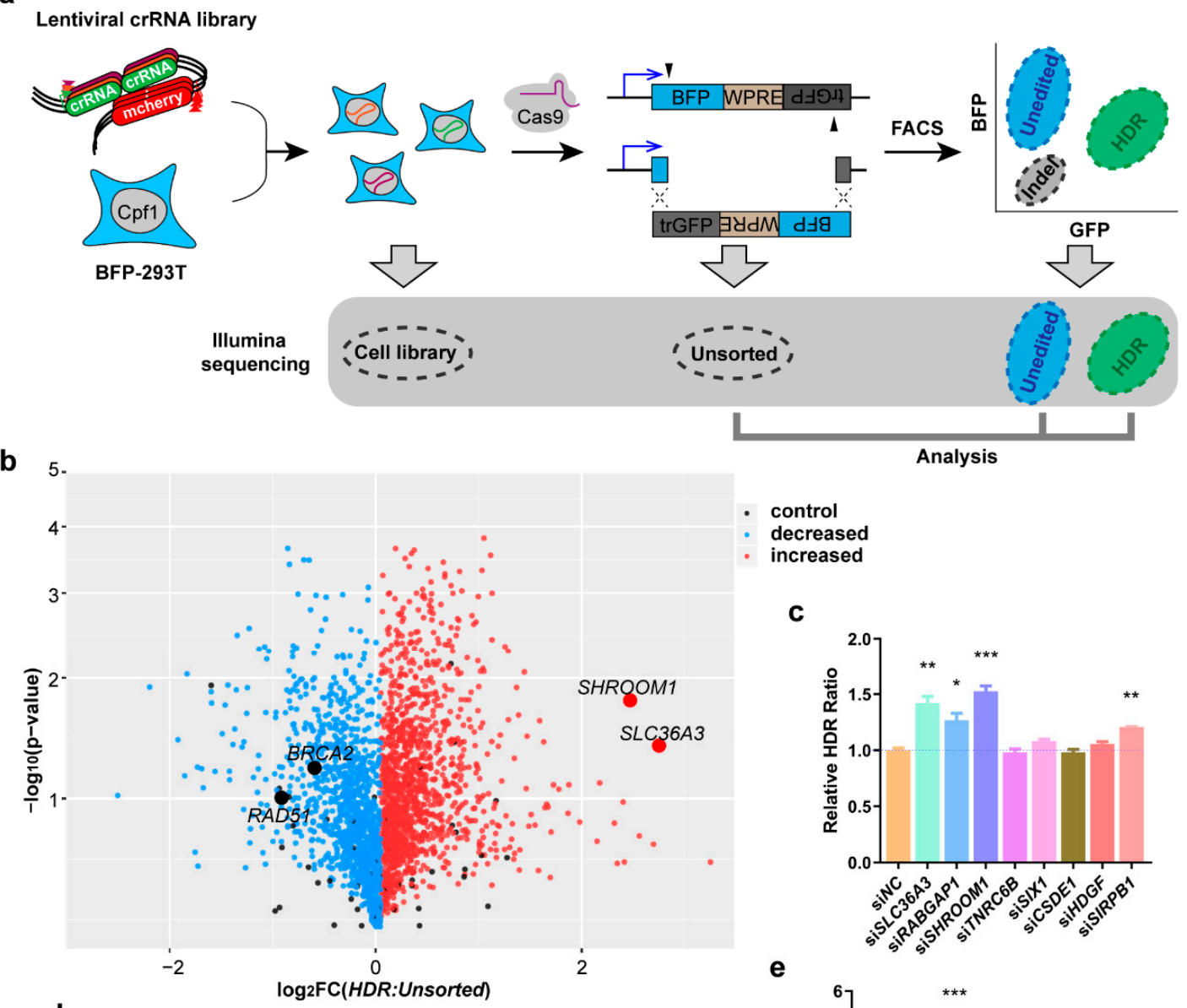

d

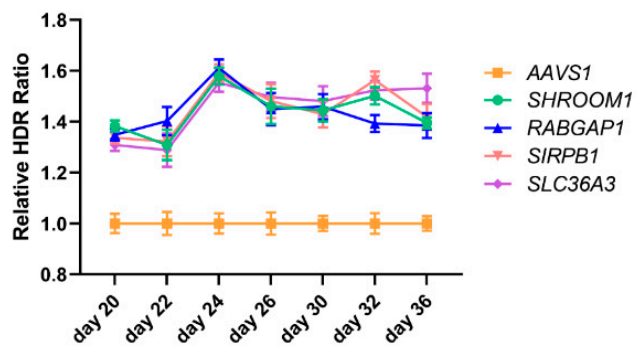

C
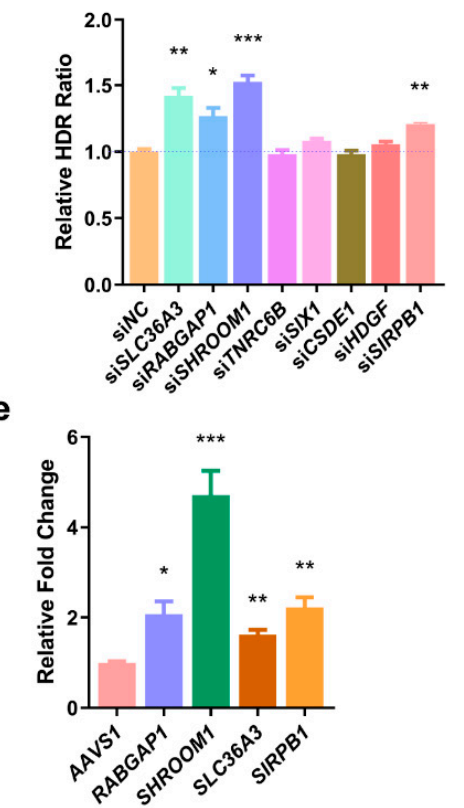

Figure 2. Paired crRNA library screening identified that SHROOM1 is an HDR suppressor of dual-cut BFP (blue fluorescence protein) reporter. (a) Schematic of paired crRNA library screening strategy in the main text; (b) multiple genes were enriched in the HDR population. Representative genes are highlighted in red. Data were generated from $n=4$ independent experiments. The $p$-value was calculated using a two-sided Student's $t$-test. (c) Relative HDR ratio of dual-cut BFP reporter cells treated with individual siRNA of top eight genes enriched in the screening. Data were generated from $n=3$ independent experiments. Error bars, $\pm \mathrm{SD}^{*} p<0.05 ;{ }^{* *} p<0.01$; ${ }^{* * *} p<0.001$ by two-sided Student's $t$-test. (d) Relative HDR ratio-time curve of dual-cut BFP reporter cell lines expressing mono-paired crRNAs. Data were collected every two days from day 20 to day 36 after cell sorting. Data were generated from $n=3$ independent experiments. (e) Genes enriched in the HDR population of simulated screening. Data were generated from $n=4$ independent experiments. Error bars, $\pm \mathrm{SD}^{*} p<0.05$; ** $p<0.01 ;{ }^{* * *} p<0.001$ by two-sided Student's $t$-test.

\subsection{Knockdown of SHROOM1 Can Promote In Vitro Genome Editing Efficiency}

We examined whether knockdown of SHROOM1 led to a more robust knock-in compared with undisturbed cells in vitro. To test this idea, we compared the HDR efficiency using siRNA as well as three types of donors: a single-strand donor (ss donor), a double-strand PCR donor (ds donor), 
and double-cut sites containing a plasmid donor (dc donor) (Figure 3a). All donors contained $800 \mathrm{bp}$ left and right homologous arms. To evaluate the knock-in efficiencies, we aimed to fuse a P2A-EGFP reporter gene to the last codon of the FBL gene in HEK293T cells. The resulting HDR efficiencies were presented as a percentage of $\mathrm{GFP}^{+}$cells (Figure 3a,b). At seven days after cell sorting HEK293T with an ss donor, sgRNA, and spCas9, the HDR efficiency of SHROOM1 siRNA $(3.65 \% \pm 0.10 \%)$ was higher than NC siRNA $(2.37 \% \pm 0.05 \%)$ (Figure 3c). Genotyping showed that SHROOM1 knockdown- mediated gene knock-in represented precise in-frame integrations, in common with the control (Figure S4a-c (Supplementary Materials)). Similarly, using a ds donor, the HDR efficiency of SHROOM1 decreased $(6.66 \% \pm 0.31 \%)$ but was still more than 3 -fold higher than that of the NC group $(2.19 \% \pm 0.23 \%)$ (Figure $3 c)$. HDR efficiency mediated by double-cut sites containing a plasmid donor was, remarkably, 10-fold higher than with classical donors [20,21]. In our study, SHROOM1 knockdown elevated HDR efficiency (though it was already at a high level) when a dc donor was used, with the knock-in efficiency increasing from $19.3 \% \pm 1.55 \%$ to $27.5 \% \pm 1.95 \%$ (Figure 3c). The similar elevated HDR ratios were generated by another siRNA of SHROOM1 (siSHROOM1') (Figure S4d (Supplementary Materials)). Therefore, SHROOM1 knockdown can significantly promote HDR efficiency at the FBL locus, whichever donor is applied (Figure 3d).

We next examined HDR efficiency at the LMNA gene locus in HEK293T (Figure S4e (Supplementary Materials)). When SHROOM1 was knocked down by siRNA, the HDR efficiency, mediated by the ds donor, increased from $29.5 \% \pm 2.00 \%$ to $36.8 \% \pm 1.71 \%$, and the HDR efficiency rose from $43.9 \% \pm 2.26 \%$ to $51.3 \% \pm 1.43 \%$ using a dc donor (Figure 3e). In human colorectal cancer cell line HCT116, the efficiency of SHROOM1 knockdown was higher than in the control when a ds donor was used at the FBL or LMNA locus (Figure 3e). The same elevation of HDR efficiency was observed at the Actb locus in mouse hepatoma cell line Hepa1-6 with different types of donors when SHROOM1 was inhibited (Figure 3f).

Together, these results indicated that the SHROOM1 knockdown method yielded a higher HDR efficiency in multiple cell lines, regardless of which type of donor was used. 


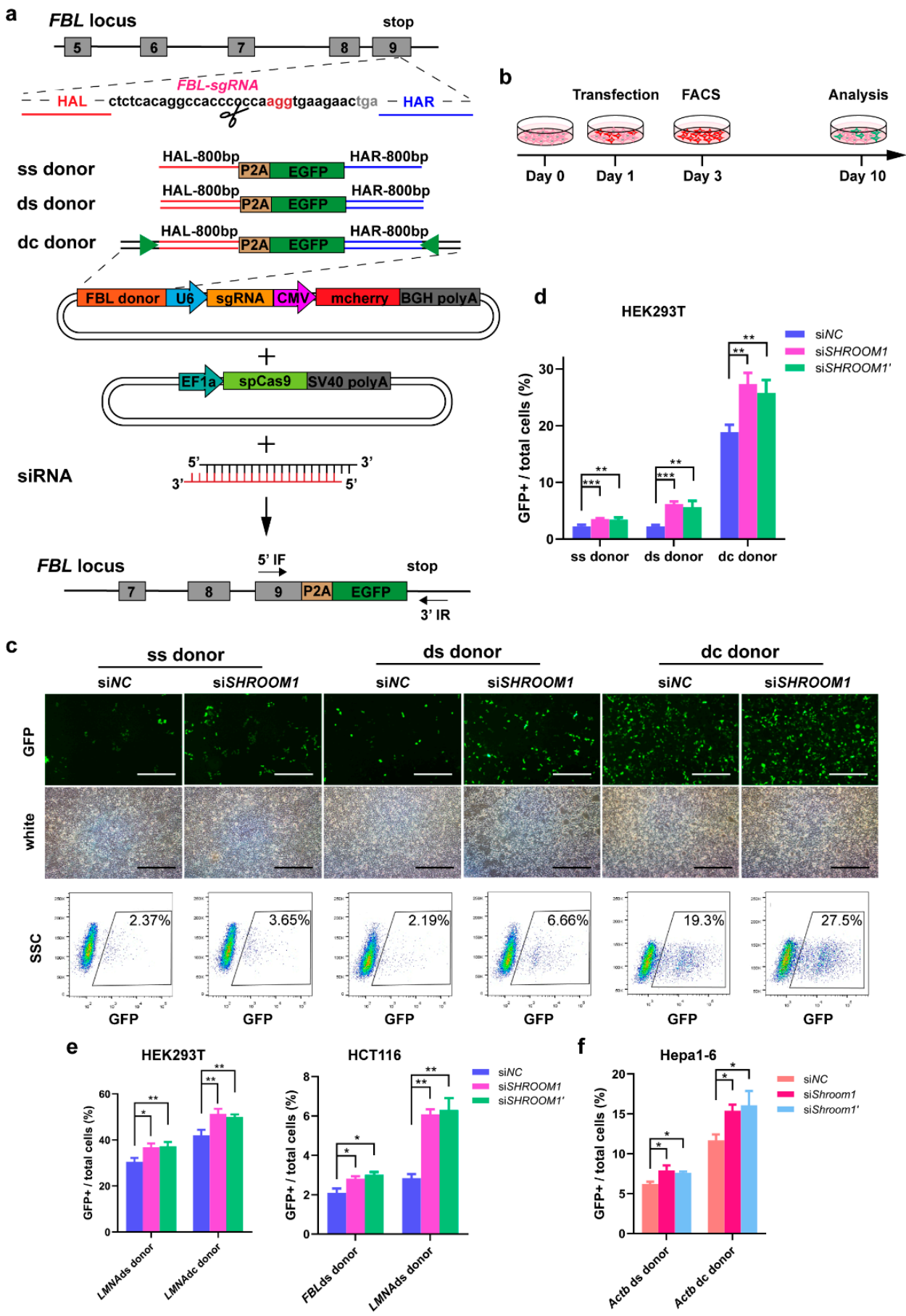

Figure 3. Knockdown of SHROOM1 enhances the knock-in efficiency of cells in vitro. (a) Schematic overview of gene-targeting strategies with siRNA and different types of donor at the $F B L$ locus. HAL/HAR, left/right homology arm; triangles, sgRNA target sites; IF/IR, inserted forward/reverse primer. ss, single strand; ds, double strand; dc, double cut. (b) Experimental scheme for targeted FBL-2A-GFP knock-in in HEK293T cells. Representative visual fields and sorting charts (c) and relative knock-in efficiency. Scale bar, $200 \mu \mathrm{m}$. (d) of ss, ds, and dc donor-based strategies with SHROOM1 siRNA or not at the FBL locus in HEK293T cells. Data were generated from $n=3$ independent experiments. Error bars, $\pm \mathrm{SD}^{* *} p<0.01 ;{ }^{* *} p<0.001$ by two-sided Student's $t$-test $(\mathbf{e}, \mathbf{f})$. Relative knock-in efficiency of ds and dc donor-based strategies with siRNA in HEK293T, HCT116, or Hepa1-6 cells. Data were generated from $n=3$ independent experiments. Error bars, $\pm \mathrm{SD}^{*} p<0.05 ;{ }^{* *} p<0.01$ by two-sided Student's $t$-test. 


\subsection{SHROOM1 Knockdown Can Promote Gene Integration in Mouse Embryos}

To investigate whether the SHROOM1 knockdown strategy could improve knock-in efficiency in generating gene-modified mice, we used the Cas9-Avidin/biotin-donor DNA system [19] and siRNA in mouse zygotes (Figure 4a and Figure S5a (Supplementary Materials)). This system has been confirmed to result in a remarkable knock-in efficiency in mouse zygotes. The high affinity of streptavidin and biotin could promote accessibility of Cas9 and the DNA donor linked to them, then local concentration of the donor at the cutting site increased and which resulted in enhanced HDR efficiency. The injected zygotes were transferred to pseudo-pregnant mice for embryo development. Genotyping of new-born mice showed that knockdown of SHROOM1 by 13.3 pg siRNA per zygote could improve precise integration efficiency from $0 \%$ to $6.5 \%$ at the $D d x 4$ locus (Figure $4 \mathrm{~b}, \mathrm{~d}$ and Figure S5b (Supplementary Materials)). A similar improvement happened at the Icos locus, from $6.7 \%$ to $12.5 \%$, with the same operation (Figure 4c,d and Figure S5b (Supplementary Materials)). Interestingly, the imprecise integration (only at the $5^{\prime}$ junction or $3^{\prime}$ junction) efficiencies of these locus also increased, accompanied by precise integration (Figure $4 b, c$ and Figure S5d (Supplementary Materials)). Therefore, knockdown of SHROOM1 could significantly promote gene integration in mouse embryos.

a

- ssDNA donor

w Cas9-Avidin mRNA

Cas9-Avic

$\mathrm{m}$ sgRNA
$=$ siRnA

Pseudo-Pregnant

Fo Tail PCR

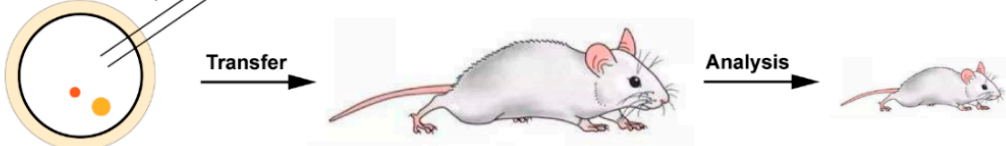

b

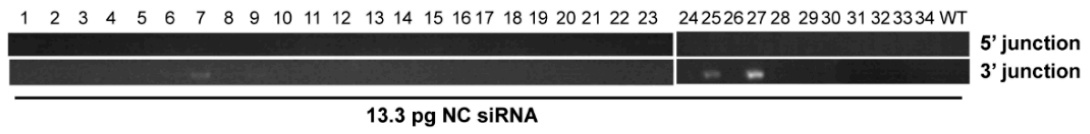

13.3 pg NC siRNA

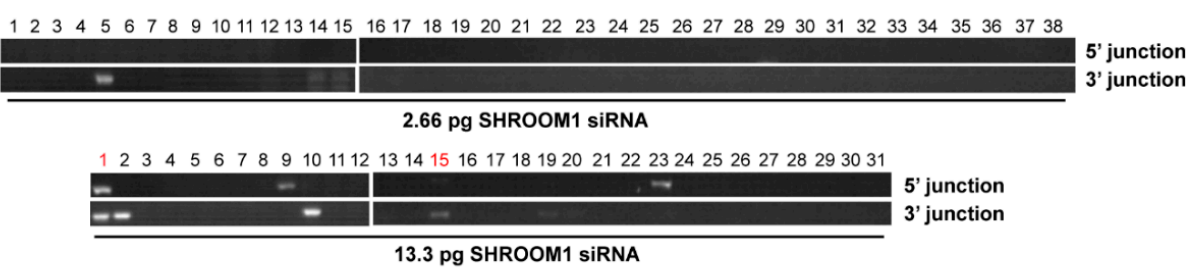

C

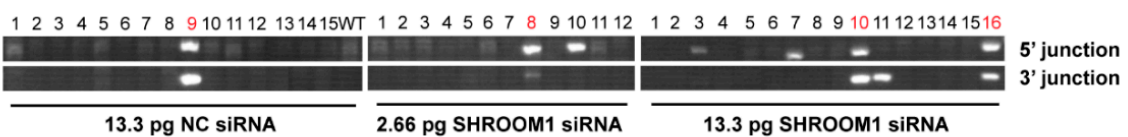

d

Summary of SHROOM1 siRNA mediated HDR at two gene loci

\begin{tabular}{cccccccccc}
\hline $\begin{array}{c}\text { Target } \\
\text { Gene }\end{array}$ & $\begin{array}{c}\text { Shroom1 } \\
\text { siRNA }\end{array}$ & $\begin{array}{c}\text { NC } \\
\text { siRNA }\end{array}$ & $\begin{array}{c}\text { Knock-in } \\
\text { Fragement }\end{array}$ & $\begin{array}{c}\text { Arm } \\
\text { length }\end{array}$ & $\begin{array}{c}\text { Numbers of } \\
\text { transferred } \\
\text { embryos }\end{array}$ & $\begin{array}{c}\text { Numbers of } \\
\text { born mice }\end{array}$ & $\begin{array}{c}\text { No } \\
\text { Insertion }\end{array}$ & $\begin{array}{c}\text { Imprecise } \\
\text { Integration }\end{array}$ & $\begin{array}{c}\text { Precise } \\
\text { Inregration }\end{array}$ \\
\hline$D d x 4$ & 0 & $13.3 \mathrm{pg}$ & $1959 \mathrm{bp}$ & $151 \mathrm{bp}$ & 115 & 34 & $31 / 34(91.2 \%)$ & $3 / 34(8.8 \%)$ & $0 / 34(0 \%)$ \\
$D d \times 4$ & $2.66 \mathrm{pg}$ & 0 & $1959 \mathrm{bp}$ & $151 \mathrm{bp}$ & 127 & 38 & $37 / 38(97.4 \%)$ & $1 / 38(2.6 \%)$ & $0 / 15(0 \%)$ \\
$D d \times 4$ & $13.3 \mathrm{pg}$ & 0 & $1959 \mathrm{bp}$ & $151 \mathrm{bp}$ & 123 & 32 & $24 / 31(77.4 \%)$ & $5 / 31(16.1 \%)$ & $2 / 31(6.5 \%)$ \\
\hline $\operatorname{lcos}$ & 0 & $13.3 \mathrm{pg}$ & $794 \mathrm{bp}$ & $128 \mathrm{bp}$ & 58 & 15 & $14 / 15(93.3 \%)$ & $0 / 15(0 \%)$ & $1 / 15(6.7 \%)$ \\
$I \cos$ & $2.66 \mathrm{pg}$ & 0 & $794 \mathrm{bp}$ & $128 \mathrm{bp}$ & 60 & 12 & $10 / 12(83.4 \%)$ & $1 / 12(8.3 \%)$ & $1 / 12(8.3 \%)$ \\
$\operatorname{lcos}$ & $13.3 \mathrm{pg}$ & 0 & $794 \mathrm{bp}$ & $128 \mathrm{bp}$ & 65 & 16 & $11 / 16(43.8 \%)$ & $3 / 16(18.8 \%)$ & $2 / 16(12.5 \%)$ \\
\hline
\end{tabular}

Figure 4. Knockdown of SHROOM1 enhances the knock-in efficiency of mouse embryos. (a) Experimental design of micro-injection. Cas9-Avidin mRNA, sgRNA, biotin-ss donor, and siRNA were injected into mouse zygotes and the injected zygotes were transferred to pseudo-pregnant mice for genotyping analysis. Genotyping of $D d x 4$ locus (b) and Icos locus (c) in mice treated with SHROOM1 siRNA or NC siRNA after incision by CRISPR/Cas9; (d) Summary of SHROOM1 siRNA-mediated HDR at the $D d x 4$ and Icos loci. Results with significant differences are highlighted in red. 


\subsection{SHROOM1 Is a Potent Suppressor of HDR in Cells}

We next explored whether the elevated knock-in efficiency was a result of the increased HDR induced by SHROOM1 inhibition. Knockout of SHROOM1 in the HEK293T cell line was constructed by SpCas 9 and two sgRNAs targeting exon 4 of the primary gene transcript (Figure 5a). Genotyping showed that there was an $815 \mathrm{bp}$ deletion and a frameshift $(+1 \mathrm{bp})$ in this $\mathrm{KO}$ cell line (Figure $5 \mathrm{a}$ and Figure S6a (Supplementary Materials)). Expression of SHROOM1 was totally abolished in KO cells (Figure $5 b$ ). Using the same knock-in strategy with a dc donor at the FBL locus, the knock-in efficiency of $\mathrm{KO}$ cells $(38.2 \% \pm 1.61 \%)$ was twice as high as in wild-type cells $(19.4 \% \pm 1.21 \%)$ (Figure $5 \mathrm{c}$ ). When YU238259, an HR inhibitor [44], was added to treat KO cells during transfection, the knock-in efficiency dropped to $23.1 \% \pm 2.21 \%$. The efficiency was not significantly different in $\mathrm{KO}$ cells treated with Scr7 (a NHEJ inhibitor [22]) (Figure 5c). After cutting by CRISPR/Cas9 and sgRNA without a donor, NHEJ was generated in HEK293T cells and could be inhibited by Scr7 (Figure S6b (Supplementary Materials)). But there is no difference of NHEJ frequency in SHROOM1 KO cells comparing with wildtype cells. Re-expression of SHROOM1 in KO cells could visibly eliminate the enhancement of knock-in efficiency (Figure 5b,c). Therefore, deletion of SHROOM1 caused a rise in knock-in efficiency through promoting HDR activity rather than NHEJ.

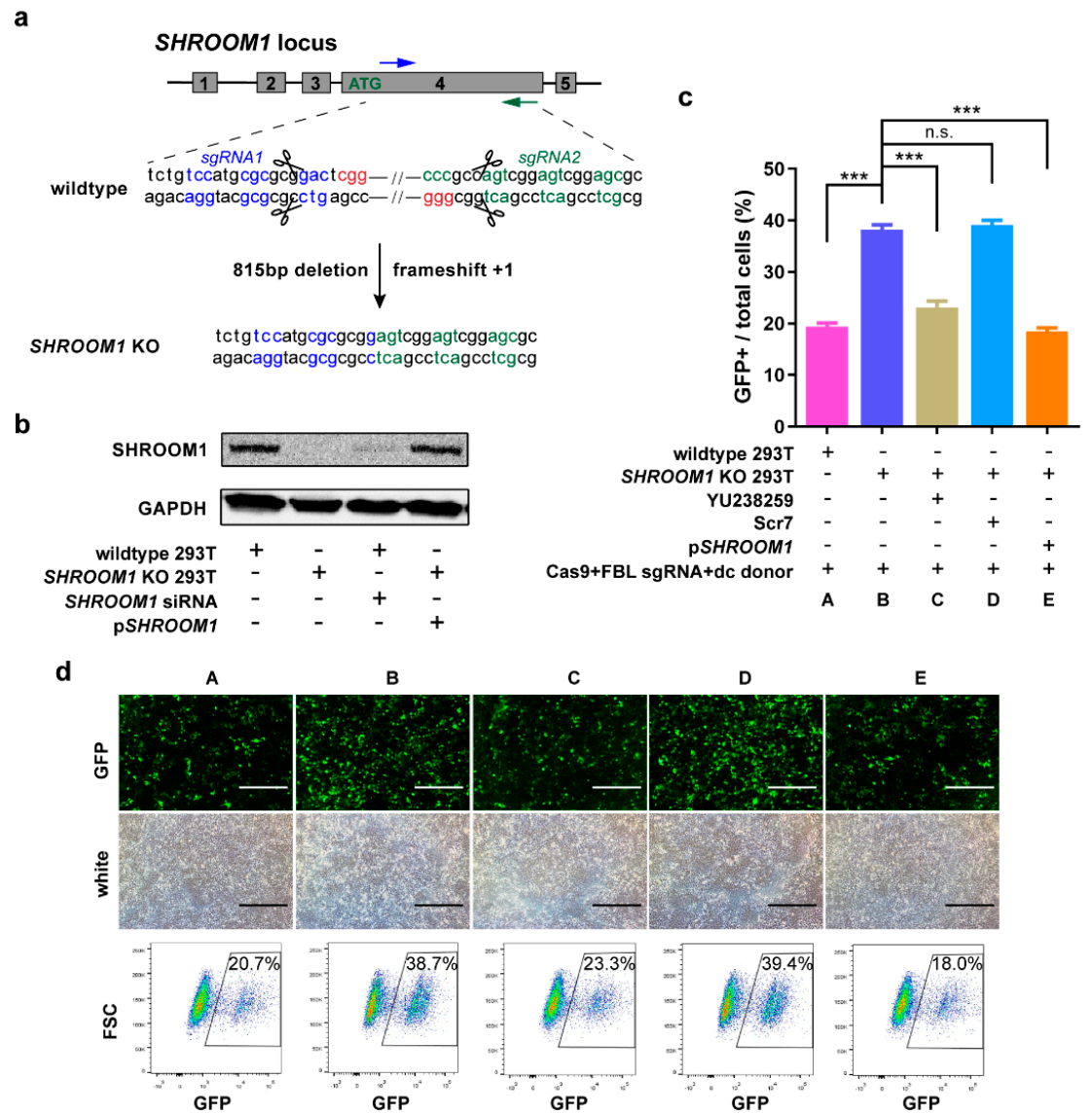

Figure 5. SHROOM1 is a potent suppressor of HDR progress. (a) Schematic of SHROOM1 deletion using CRISPR/Cas9 and two sgRNAs in HEK293T cells. PAM, highlighted in red; protein codon, highlighted in blue or green, KO for knockout; (b) Western blot of different types of cells or treatment. KO, for knockout; pSHROOM1 for SHROOM1 cDNA contained plasmid; relative knock-in efficiency (c) and representative visual fields and sorting charts (d) in SHROOM1 knockout or wild-type HEK293T cells with treatments. YU238259, an HR inhibitor; Scr7, a NHEJ inhibitor; dc, double-cut sites contained donor. Data were generated from $n=3$ independent experiments. Error bars, $\pm \mathrm{SD}^{* * *} p<0.001$; n.s., no significance; by two-sided Student's $t$-test. Scale bar, $200 \mu \mathrm{m}$. 


\section{Discussion}

In summary, through a genome-wide screening and following paired crRNA library screening with CRISPR-Cpf1, we have identified SHROOM1 as a potent suppressor of HDR in BFP reporter cells. Knockdown of SHROOM1 can improve the knock-in efficiency in both cultured mammalian cells and mouse embryos.

Genome-wide screening in iDR-GFP U2OS by siRNAs exhibits high stability and accuracy. Many genes appear in our sight, such as CtIP and BRCA1, and may play an important role in HDR maintaining activity. In order to exclude potential false positive and find potent HDR-suppressing genes, we use CRIPSR/Cpf1 and paired crRNAs to implement the next screening. Considering the instability and lower HDR incident of the BFP-reporter after transfecting the CRISPR system in U2OS, we designed a dual-cut BFP reporter in HEK293T to carry on the subsequent screening. Finally, we found suppressing SHROOM1 could improve the HDR ratio in dual-cut BFP 293T significantly. In common with SHROOM1, inhibition of SLC36A3 shows similar ability to enhance the HDR efficiency in siRNA-treated cells (Figure 2c) or stable cell line expressing paired crRNA and Cpf1 (Figure 2d). But the parallelly increased ratios may not completely represent the ability to promote HDR, because it was generated in cells with heterogeneity when transfected and background HDR frequency and edited for two days. Higher statistical stability of SHROOM1 $(5.55 \pm 1.53, p=0.016)$ shown in CRISPR-Cpf1 screening (Figure $2 \mathrm{~b}$ and Table S5 (Supplementary Materials)) may cause remarkable enrichment in the scaled-down screening lasting for seven days comparing to $\operatorname{SLC} 36 A 3 \operatorname{KD}(6.74 \pm 3.70, p=0.041)$ (Figure 2e).

Our work shows that HDR inhibition or re-expression of SHROOM1 can eliminate the improvement of knock-in efficiency in KO cells, which indicates that deletion of SHROOM1 allows cellular HDR activities to raise the gene editing efficiency. Though NHEJ is the main damage repair approach [45], it is not suitable for use with SHROOM1. In SHROOM1-KO HEK293T cells, the NHEJ frequency shows no difference with wildtype. SHROOM1 has been reported to involve in the assembly of microtubule arrays during cell elongation. But the mechanism of SHROOM1 regulating HDR still remains unknown. Side scatter (SSC) represents the complexity of the cell in the flow cytometer, and forward scatter (FSC) represents cell size. We found that deficiency of SHROOM1 showed higher SSC and FSC value, which represents increased intracellular complexity and cell size of KO cells (Figure S6c (Supplementary Materials)). This is a foreseeable result because of the function of SHROOM1 in the cytoskeleton. We supposed the altered complexity and size might affect the cell cycle to regulate HDR activity which occurs at the late S/G2 phase. Wildtype and SHROOM1 KO HEK293T were stained with propidium iodide (PI, a DNA dye which fluorescence is direct interrelated with binding DNA amount) and then analyzed by the flow cytometer to detect the possible change of cell numbers at different phases. It is depressing that there is no difference of cell cycle when SHROOM1 is absent (Figure S6d (Supplementary Materials)). Therefore, other methods like target searching or interaction analysis to find the functional pathway may provide some clues to uncover the mechanism that SHROOM1 negatively regulates HDR.

Precision genome editing by modified nucleases such as the CRISPR-Cas9 system can be enhanced by the optimization of the donor, modulating the cellular DNA repair machine or selection of damage repair approaches. We demonstrated that inhibition of SHROOM1 can promote cellular knock-in efficiency using different types of donors. Compared with other strategies such as expressing an HDR-promoted protein, adding the siRNA of SHROOM1 has more advantages because of its smaller size and low cost. Altogether, our work establishes that inhibiting SHROOM1 is a robust tool for enhancing CRISPR-mediated precision genome editing in human cells and mouse embryos, thereby aiding the study of gene function and modeling of human diseases. 


\section{Methods}

\subsection{Construction of Plasmids}

To construct the rtTA-expressing lentiviral vector, an EF1 $\alpha$ promoter, rtTA, and the IRES-connected neomycin were subcloned into the cloning site between cPPT/CTS and $3^{\prime}$ LTR of a modified pLKO vector (no. 13425, Addgene, Watertown, MA, USA).

To construct the I-SceI induced expressing lentiviral vector, TRE-miniCMV-I-SceI expression cassettes and a PGK-puromycin expression cassette were subcloned into the cloning site between cPPT/CTS and 3' LTR of a modified pLKO vector (no. 13425, Addgene, Watertown, MA, USA).

To construct the HDR reporter lentiviral vector, CMV-SceGFP expression cassettes, a PGK-hygromycin expression cassette, and an interval GFP fragment were subcloned into the cloning site between CPPT/CTS and $3^{\prime}$ LTR of a modified pLKO vector (no. 13425, Addgene, Watertown, MA, USA).

To construct the Cpf1 expression lentiviral vector, a CMV-Cpf1-P2A-puromycin expression cassette was subcloned into the cloning site between cPPT/CTS and 3' LTR of a modified pLKO vector (no. 13425, Addgene, Watertown, MA, USA).

To construct the dual-cut BFP reporter lentiviral vector, an EF1 $\alpha$-BFP expression cassette, WPRE, and truncated GFP were subcloned into the cloning site between cPPT/CTS and 3' LTR of a modified pLKO vector (no. 13425, Addgene, Watertown, MA, USA).

To generate a single paired crRNA expression lentiviral vector, a U6 promoter, two BsmBI sites, and a CMV-mcherry expression cassette were subcloned into the cloning site between cPPT/CTS and 3' LTR of a modified pLKO vector (no. 13425, Addgene, Watertown, MA, USA).

To construct the double-cut sites donor for the FBL/LMNA/Actb gene, donor DNA (800 bp HAL-p2A-mGFP-800bp HAR) sandwiched by a 23-nt $A c t b$-sgRNA target sequence, U6- $A c t b$-sgRNA expression cassette, and CMV-mcherry expression cassette was subcloned into the cloning site of a modified pcDNA3.1+ vector (V79020, Invitrogen-Thermo Fisher Scientific, Waltham, MA, USA).

To construct the SHROOM1 expression vector, SHROOM1 cDNA was subcloned into the cloning site of a pcDNA3.1+ vector (V79020, Invitrogen-Thermo Fisher Scientific, Waltham, MA, USA).

Primary gene and donor sequences are given in Table S1 (Supplementary Materials).

\subsection{Cell Culture and Cell Lines}

U2OS, HEK293T, HCT116, and Hepa1-6 cells were obtained from the American Type Culture Collection (ATCC, Manassas, VA, USA). Cell lines were cultured in DMEM supplemented with 10\% fetal bovine growth serum (SH30084.03, Hyclone-Thermo Fisher Scientific, Waltham, MA, USA) and 1× penicillin-streptomycin (10378016, GIBCO-Thermo Fisher Scientific, Waltham, MA, USA). Cells were grown at $37^{\circ} \mathrm{C}$ with $5 \% \mathrm{CO}_{2}$. To generate cell lines expressing the induced DR-GFP reporter, U2OS cells were transduced with lentiviruses (multiplicity of infection $[\mathrm{MOI}]=0.1$ ) carrying the EF1 $\alpha$-rtTA expression cassette, TRE-miniCMV-I SceI expression cassette, and CMV-SceGFP expression cassette successively. G418 $(1 \mathrm{mg} / \mathrm{mL})$, puromycin $(1.25 \mu \mathrm{g} / \mathrm{mL})$, or hygromycin $(1 \mathrm{mg} / \mathrm{mL})$ was used to select the corresponding stable cells. For generating dual-cut BFP reporter cells, HEK293T cells were transduced with lentiviruses (multiplicity of infection $[\mathrm{MOI}]=0.1$ ) carrying the CMV-Cpf1-P2A-puromycin expression cassette, then selected by puromycin $(1.25 \mu \mathrm{g} / \mathrm{mL})$. Subsequently, the stable cells were transduced with lentiviruses (multiplicity of infection $[\mathrm{MOI}]=0.1$ ) carrying a dual-cut BFP reporter and sorted by flow cytometry to produce a pure population of dual-cut BFP reporter cells (BFP-293T). To generate single paired crRNA expressed library cells, BFP-293T cells were transduced with lentiviruses (multiplicity of infection $[\mathrm{MOI}]=0.1$ ) to carry U6-paired crRNA and CMV-mcherry expression cassettes and sorted by flow cytometry.

\subsection{Genome-Wide Screening}

First, 2000 iDR-GFP U2OS cells were planted in 384-well plates. Eighteen hours later, $0.15 \mu \mathrm{L}$ RNAiMAX (catalog 13778150, Invitrogen-Thermo Fisher Scientific, Waltham, MA, USA) and 2 pmol 
siRNAs (candidate or positive control, Genepharma, Suzhou, China) were transferred into each well by the Agilent (Santa Clara, CA, USA) Bravo automated liquid handling platform. Forty-eight hours after transfection, cells were treated with $2 \mu \mathrm{g} / \mathrm{mL}$ doxycycline (D8960, Solarbio, Beijing, China) for two days to induce DSB and HDR. Individual wells in plates were immobilized and stained by DAPI. Then, fluorescent data were collected by an Image Xpress instrument (Molecular Devices, San Jose, CA, USA) and processed by Meta Xpress. Ratio $=10 \times$ No. $\left(\mathrm{GFP}^{+}\right.$cells)/No. (Total cells), FC $=$Ratio (siRNA)/Ratio (NC).

\subsection{Genomic DNA Extraction and PCR Amplification for Editing Region}

Seventy-five percent of the edited cells ( $\sim 500,000$ cells) were collected every two days from day 20 to day 38 after cell sorting and washed once in a PBS buffer solution. Genome extractions were carried out according to the instructions of the kit (DP304, Tiangen, Beijing, China) and eluted with $80 \mu \mathrm{L}$ distilled water for downstream analysis. Amplification of edited loci was performed with the locus-specific primer pairs described in Table S2 (Supplementary Materials) using $2 \times$ Q5 master mix (M0494L, New England Biolabs, Ipswich, MA, USA) and $200 \mathrm{ng}$ of genomic DNA. The thermocycler was set for one cycle at $98^{\circ} \mathrm{C}$ for $30 \mathrm{~s}, 35$ cycles at $98^{\circ} \mathrm{C}$ for $15 \mathrm{~s}, 60^{\circ} \mathrm{C}$ for $15 \mathrm{~s}$, and $72{ }^{\circ} \mathrm{C}$ for $10 \mathrm{~s}$, respectively, and one cycle at $72{ }^{\circ} \mathrm{C}$ for $1 \mathrm{~min}$, and held at $4{ }^{\circ} \mathrm{C}$. PCR amplicons were run on a $1.5 \%$ agarose gel to verify the size and purity, and quantified by nanodrop. The resulting DNA was used for direct analysis or reamplified with primers containing Illumina adaptors.

\subsection{Next-Generation Sequencing (NGS) Library Generation and Sequencing}

One hundred nanograms of purified PCR amplicons were used as a library template. The sequencing library was obtained from the replicates using a NEBNext Ultra II RNA Kit (E7775S, New England Biolabs, Ipswich, MA, USA). Pooled samples were purified with SPRI beads. Library size and purity was verified by Agilent 2100 before sequencing on a Nova seq (Illumina, San Diego, CA, USA) using a Reagent Kit S2 (Novaseq 6000, Illumina) $(2 \times 150$ bp).

\subsection{Pooled Screen}

After culturing for 28 days, one $1 \times 10^{7}$ aliquot of single paired crRNA expressed library cells was collected for sequencing of library quality control, a second $1 \times 10^{7}$ cell aliquot was collected for transfection, and the remaining cell libraries were frozen. Plasmid DNA ( $2 \mu \mathrm{g}$ SpCas9 and $1.2 \mu \mathrm{g}$ sgRNA) and lipofectamine $2000(6 \mu \mathrm{L})$ were incubated together and transfected into $3 \times 10^{6}$ cells cultured in a single well of a six-well plate. The transfected cell libraries were cultured for seven days. A $1 \times 10^{7}$ cell aliquot was collected from the transfected cell library (Unsorted), and $2 \times 10^{7}$ cells were sorted into $\mathrm{BFP}^{+}$(Unedited) and $\mathrm{GFP}^{+}$(HDR) populations. The collected cell populations were rinsed in PBS and frozen at $-80^{\circ} \mathrm{C}$. DNA from each cell population-unsorted, unedited, and HDR-was purified with genome purification kits (DP304, Tiangen, Beijing, China) and the total amount of DNA was quantified. A maximum of $1 \mu \mathrm{g}$ of genomic DNA was amplified in a single KAPA HiFi PCR (KK2602, KAPA Biosystems-Roche, Pleasanton, CA, USA) reaction using primers specific to the crRNA cassette. Up to 24 PCR reactions were set up for each cell population to obtain the desired coverage of the cell library. The thermocycler was set for one cycle of $98^{\circ} \mathrm{C}$ for $30 \mathrm{~s}, 23$ cycles of $98{ }^{\circ} \mathrm{C}$ for $20 \mathrm{~s}$, $56^{\circ} \mathrm{C}$ for $15 \mathrm{~s}$, and $72{ }^{\circ} \mathrm{C}$ for $25 \mathrm{~s}$, respectively, and one cycle of $72{ }^{\circ} \mathrm{C}$ for $5 \mathrm{~min}$. PCR reactions were pooled and run on 1.5\% agarose gel and purified by a gel extraction kit (28706, Qiagen, Germantown, MD, Germany). Amplified DNA from each cell population was normalized to input cell numbers and prepared for sequencing as mentioned above.

\subsection{Pooled Screen Analysis}

Sequence reads were trimmed, aligned to crRNA sequence templates, and quantified. Read counts for each crRNA pairs were normalized and compared to the distribution of untargeted control guides to determine the significance and $\log _{2}$ magnitude of change. 


\section{8. $q P C R$}

For qPCR, 100,000-200,000 cells were collected and RNA extracted with the TRNzol (DP424, Tiangen Beijing, China). cDNA was produced from $1 \mu \mathrm{g}$ of purified RNA using the FastKing RT Kit (KR116, Tiangen Beijing, China). qPCR reactions were performed with the SuperReal SYBR Green PreMix Plus (FP205, Tiangen Beijing, China) in a total volume of $10 \mu \mathrm{L}$, with primers at final concentrations of $500 \mathrm{nM}$. The thermocycler was set for one cycle of $95^{\circ} \mathrm{C}$ for $15 \mathrm{~min}$, and 40 cycles of $95^{\circ} \mathrm{C}$ for $10 \mathrm{~s}, 60^{\circ} \mathrm{C}$ for $20 \mathrm{~s}$, and $72{ }^{\circ} \mathrm{C}$ for $32 \mathrm{~s}$, respectively. Fold enrichment of the assayed genes over the control GAPDH loci was calculated using the $2^{-\triangle \Delta C t}$ method.

\section{9. siRNA Interference and HDR Assays with Dual-Cut BFP Reporter}

Dual-cut BFP 293T cells were seeded at 60-70\% confluency into 24-well plates. For qPCR, siRNA (500 ng, Table S3 (Supplementary Materials)) was transfected individually using lipofectamine 2000 $(1 \mu \mathrm{L})$ and harvested two days after transfection. For HDR assays, a cocktail of siRNA (500 ng), SpCas9 (800 ng), and sgRNA (400 ng) was transfected using lipofectamine $2000(2.5 \mu \mathrm{L})$. Enriched paired crRNA stably expressed dual-cut BFP 293T cells were seeded in the same manner described above and transfected with SpCas9 (800 ng) and sgRNA (400 ng) for HDR assays. The cells were collected three days after transfection for analyzing by flow cytometry for $\mathrm{GFP}^{+}$cells using a LSRFortessa (Becton, Dickinson and Company, San Jose, CA, USA).

\subsection{In Vitro Gene Knock-in}

HEK293T, HCT116, Hepa1-6, and SHROOM1 knockout HEK293T cells were seeded at 60-70\% confluency into 12-well plates. For the ss donor or ds donor, these cells were transfected with donor (800 ng), SpCas9 (1200 ng), sgRNA (600 ng, Table S3 (Supplementary Materials)), and siRNA (1000 ng) using lipofectamine $2000(8 \mu \mathrm{L})$. For the dc donor, cells were transfected with donor-sgRNA cassette (1200 ng), SpCas9 (1200 ng), and siRNA (1000 ng). Modified cells were collected two days after transfection and sorted by flow cytometry for mcherry ${ }^{+}$cells using a MoFlo (Beckman Coulter, Brea, CA, USA). Seven days after sorting, the ratio of $\mathrm{GFP}^{+}$cells was analyzed using a LSRFortessa (Becton, Dickinson and Company, San Jose, CA, USA) (Figure 4b). Genotyping of GFP+ cells was conducted using primers in Table S2.

For the comparison of different knock-in strategies, treatment with $5 \mu \mathrm{M}$ YU238259 (S8379, Selleck Chemicals, Houston, TX, USA) and $1 \mu$ M Scr7 (S7742, Selleck Chemicals, Houston, TX, USA) was started one day before transfection and was continued until two days after transfection. The SHROOM1-expressed plasmid (1000 ng) was co-transfected with the components above into $\mathrm{KO}$ cells.

\subsection{Western Blotting}

Primary antibodies against the following proteins were used: SHROOM1 (bs-13735R; Bioss, Beijing, China); GAPDH (sc-365062; Santa Cruz, Dallas, TX, USA). For each protein antibody, the manufacturer's recommended dilutions were used. Mouse or rabbit immunoglobulin G was visualized with the following HRP-conjugated secondaries at a 1:5000 dilution: horse anti-mouse (\# 7076S, Cell Signaling Technology, Danvers, MA, USA); goat anti-rabbit (\# 7074S, Cell Signaling Technology, Danvers, MA, USA). The gray-scale value was analyzed by ImageJ software.

\subsection{Micro-Injection and Genotyping}

$D d x 4$ and Icos targets were designed according to the protocol described at http://crispr.mit.edu. The cleavage efficiency was measured using in vitro detection assay (VK-007, ViewSolid, Beijing, China). The genomic sequences were amplified and purified, and then used as cutting templates for each sgRNA. For each locus, four targets were designed and their efficiencies measured, and the most efficient target was selected (Table S3 (Supplementary Materials)). All animal procedures were performed 
strictly according to the Animal Care Guidelines. Fertilized zygotes were collected from ICR mouse

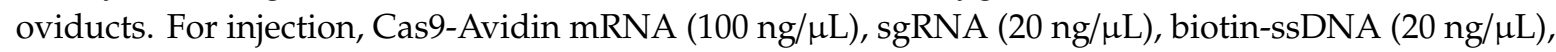
and siRNA $(1.33 \mu \mathrm{g} / \mu \mathrm{L})$ (Table S3 (Supplementary Materials)) were mixed, and then injected into zygotes. The injected zygotes were first cultured in KSOM (M1450, Easycheck, Nanjing, China) with FBS at $37^{\circ} \mathrm{C}$ and $5 \% \mathrm{CO}_{2}$, and then transferred into pseudo-pregnant female ICR mice. Mouse tails were lysed at $55^{\circ} \mathrm{C}$ with proteinase $\mathrm{K}$ overnight. Genomic DNA were extracted from $\mathrm{F}_{0}$ tails and amplified by MightyAmp (074A, Takara, Kusatsu, Shig, Japan). Two pairs of primers were designed for the $5^{\prime}$ junction and $3^{\prime}$ junction for detecting the precise gene insertion (Figure S5a and Table S3 (Supplementary Materials)). After adding A at the $3^{\prime}$ terminal, they were ligased into a $\mathrm{T}$ vector for further sequencing.

\subsection{Cell Cycle Analysis}

Cells were seeded in a 6-well tissue culture plate $(4 \times 105$ cells/well $)$ and digested with $0.05 \%$ trypsin. After treatment, the cells were collected and washed with PBS. RNase A solution $(100 \mu \mathrm{L})$ was added, and cells were incubated for $30 \mathrm{~min}$ at $37^{\circ} \mathrm{C}$. Finally, $400 \mu \mathrm{L}$ PI (P8080-10 mg, Solarbio, Beijing, China) was added and incubated for $30 \mathrm{~min}$ at room temperature. The DNA content was detected by flow cytometry. The data were analyzed by LSRFortessa (Becton, Dickinson and Company, San Jose, CA, USA). The percentage of cells in the G1 phase, the S phase, and the G2 phase were analyzed.

Supplementary Materials: The following are available online at http://www.mdpi.com/1422-0067/21/16/5821/s1. Figure S1: Construction of iDR-U2OS reporter cells. Figure S2. Performances of paired crRNAs and the dual-cut BFP reporter guarantees feasibility of the screening. Figure S3. Validation of the representative genes demonstrates the reliability of crRNA screening in dual-cut BFP reporter cells. Figure S4. Knockdown of SHROOM1 mediates precise target editing after incision by CRISPR/Cas9 in vitro. Figure S5. Knockdown of SHROOM1 promotes precise target editing after incision by CRISPR/Cas9 in mouse embryos. Table S1: Gene sequences used in this study $\left(5^{\prime}-3^{\prime}\right)$. Table S2. Primer sequences used in this study $\left(5^{\prime}-3^{\prime}\right)$. Table S3. siRNA, sgRNA target and crRNA target sequences used in this study $\left(5^{\prime}-3^{\prime}\right)$. Table S4. Genome wide screening results. Table S5. CRISPR screening gene list and screening results. Table S6. All values used in figures.

Author Contributions: Z.Z. performed most of the experiments. H.Z. did the genome-wide screening. T.X. helped with the design of the crRNA library and sequence analysis. J.W. and D.Y. did the high-throughput screening. D.Z. did the synthesis of the oligo library. J.L. did the high-throughput sequencing. Y.Y. did the optimization of in vitro knock-in targets. C.S. provided the mice and micro-injection platform. Y.Z. helped with part of the flow cytometer analysis. Z.Z. and J.J.X. designed the experiments and wrote the manuscript. All authors have read and agreed to the published version of the manuscript.

Funding: This research was supported by the National Natural Science Foundation of China (81827809 to Jianzhong $\mathrm{Xi}, 81421004$ to Jianzhong $\mathrm{Xi}$ ).

Acknowledgments: We thank the flow cytometry Core at National Center for Protein Sciences at Peking University, particularly Du Liying and Lv Hongxia, for technical help.

Conflicts of Interest: The authors declare no competing financial interests.

\section{Abbreviations}

$\begin{array}{ll}\text { SiRNA } & \text { Small interfering RNA } \\ \text { BRCA2 } & \text { Breast Cancer Type 2 Susceptibility Protein } \\ \text { BRCA1 } & \text { Breast Cancer Type 1 Susceptibility Protein } \\ \text { RAD51 } & \text { DNA Repair Protein RAD51 Homolog 1 } \\ \text { SSTR3 } & \text { Somatostatin Receptor Type 3 } \\ \text { SLC36A3 } & \text { Proton-coupled Amino Acid Transporter 3 } \\ \text { CtIP } & \text { DNA endonuclease RBBP8 } \\ \text { SHROOM1 } & \text { Protein Shroom1 } \\ \text { UPF1 } & \text { Regulator of nonsense transcripts 1 } \\ \text { AAVS1 } & \text { Adeno-associated Virus Integration Site 1 } \\ \text { FBL } & \text { rRNA 2'-O-methyltransferase Fibrillarin } \\ \text { LMNA } & \text { Prelamin-A/C } \\ \text { Actin } & \text { Actin, cytoplasmic 1 } \\ \text { Ddx4 } & \text { ATP-dependent RNA Helicase DDX4 } \\ \text { Icos } & \text { Inducible T-cell Costimulator } \\ \text { GAPDH } & \text { Glyceraldehyde 3-Phosphate Dehydrogenase }\end{array}$




\section{References}

1. Hockemeyer, D.; Soldner, F.; Beard, C.; Gao, Q.; Mitalipova, M.; DeKelver, R.C.; Katibah, G.E.; Amora, R.; Boydston, E.A.; Zeitler, B.; et al. Efficient targeting of expressed and silent genes in human ESCs and iPSCs using zinc-finger nucleases. Nat. Biotechnol. 2009, 27, 851-857. [CrossRef]

2. Hockemeyer, D.; Wang, H.; Kiani, S.; Lai, C.S.; Gao, Q.; Cassady, J.P.; Cost, G.J.; Zhang, L.; Santiago, Y.; Miller, J.C.; et al. Genetic engineering of human pluripotent cells using TALE nucleases. Nat. Biotechnol. 2011, 29, 731-734. [CrossRef]

3. Yang, H.; Wang, H.; Shivalila, C.S.; Cheng, A.W.; Shi, L.; Jaenisch, R. One-step generation of mice carrying reporter and conditional alleles by CRISPR/Cas-mediated genome engineering. Cell 2013, 154, 1370-1379. [CrossRef]

4. Kim, Y.G.; Cha, J.; Chandrasegaran, S. Hybrid restriction enzymes: Zinc finger fusions to Fok I cleavage domain. Proc. Natl. Acad. Sci. USA 1996, 93, 1156-1160. [CrossRef]

5. Porteus, M.H.; Baltimore, D. Chimeric nucleases stimulate gene targeting in human cells. Science 2003, $300,763$. [CrossRef]

6. Christian, M.; Cermak, T.; Doyle, E.L.; Schmidt, C.; Zhang, F.; Hummel, A.; Bogdanove, A.J.; Voytas, D.F. Targeting DNA double-strand breaks with TAL effector nucleases. Genetics 2010, 186, 757-761. [CrossRef] [PubMed]

7. Jinek, M.; Chylinski, K.; Fonfara, I.; Hauer, M.; Doudna, J.A.; Charpentier, E. A programmable dual-RNA-guided DNA endonuclease in adaptive bacterial immunity. Science 2012, 337, 816-821. [CrossRef] [PubMed]

8. Cong, L.; Ran, F.A.; Cox, D.; Lin, S.; Barretto, R.; Habib, N.; Hsu, P.D.; Wu, X.; Jiang, W.; Marraffini, L.A.; et al. Multiplex genome engineering using CRISPR/Cas systems. Science 2013, 339, 819-823. [CrossRef] [PubMed]

9. Zetsche, B.; Gootenberg, J.S.; Abudayyeh, O.O.; Slaymaker, I.M.; Makarova, K.S.; Essletzbichler, P.; Volz, S.E.; Joung, J.; van der Oost, J.; Regev, A.; et al. Cpf1 is a single RNA-guided endonuclease of a class 2 CRISPR-Cas system. Cell 2015, 163, 759-771. [CrossRef] [PubMed]

10. Kim, D.; Kim, J.; Hur, J.K.; Been, K.W.; Yoon, S.H.; Kim, J.S. Genome-wide analysis reveals specificities of Cpf1 endonucleases in human cells. Nat. Biotechnol. 2016, 34, 863-868. [CrossRef] [PubMed]

11. Maresca, M.; Lin, V.G.; Guo, N.; Yang, Y. Obligate ligation-gated recombination (ObLiGaRe): Custom-designed nuclease-mediated targeted integration through nonhomologous end joining. Genome Res. 2013, 23, 539-546. [CrossRef] [PubMed]

12. Suzuki, K.; Tsunekawa, Y.; Hernandez-Benitez, R.; Wu, J.; Zhu, J.; Kim, E.J.; Hatanaka, F.; Yamamoto, M.; Araoka, T.; Li, Z.; et al. In vivo genome editing via CRISPR/Cas9 mediated homology-independent targeted integration. Nature 2016, 540, 144-149. [CrossRef] [PubMed]

13. Nakade, S.; Tsubota, T.; Sakane, Y.; Kume, S.; Sakamoto, N.; Obara, M.; Daimon, T.; Sezutsu, H.; Yamamoto, T.; Sakuma, T.; et al. Microhomology-mediated end-joining-dependent integration of donor DNA in cells and animals using TALENs and CRISPR/Cas9. Nat. Commun. 2014, 5, 5560. [CrossRef] [PubMed]

14. Iyer, S.; Suresh, S.; Guo, D.; Daman, K.; Chen, J.C.J.; Liu, P.; Zieger, M.; Luk, K.; Roscoe, B.P.; Mueller, C.; et al. Precise therapeutic gene correction by a simple nuclease-induced double-stranded break. Nature 2019, 568, 561-565. [CrossRef] [PubMed]

15. Cox, D.B.; Platt, R.J.; Zhang, F. Therapeutic genome editing: Prospects and challenges. Nat. Med. 2015, 21, 121-131. [CrossRef]

16. Maruyama, T.; Dougan, S.K.; Truttmann, M.C.; Bilate, A.M.; Ingram, J.R.; Ploegh, H.L. Increasing the efficiency of precise genome editing with CRISPR-Cas9 by inhibition of nonhomologous end joining. Nat. Biotechnol. 2015, 33, 538-542. [CrossRef]

17. Gutierrez-Triana, J.A.; Tavhelidse, T.; Thumberger, T.; Thomas, I.; Wittbrodt, B.; Kellner, T.; Anlas, K.; Tsingos, E.; Wittbrodt, J. Efficient single-copy HDR by 5' modified long dsDNA donors. eLife 2018, 7, e39468. [CrossRef]

18. Yu, Y.; Guo, Y.; Tian, Q.; Lan, Y.; Yeh, H.; Zhang, M.; Tasan, I.; Jain, S.; Zhao, H. An efficient gene knock-in strategy using 5'-modified double-stranded DNA donors with short homology arms. Nat. Chem. Biol. 2020, 16, 387-390. [CrossRef] 
19. Ma, M.; Zhuang, F.; Hu, X.; Wang, B.; Wen, X.Z.; Ji, J.F.; Xi, J.J. Efficient generation of mice carrying homozygous double-floxp alleles using the Cas9-Avidin/Biotin-donor DNA system. Cell Res. 2017, 27, 578-581. [CrossRef]

20. Yao, X.; Wang, X.; Hu, X.; Liu, Z.; Liu, J.; Zhou, H.; Shen, X.; Wei, Y.; Huang, Z.; Ying, W.; et al. Homology-mediated end joining-based targeted integration using CRISPR/Cas9. Cell Res. 2017, 27, 801-814. [CrossRef]

21. Zhang, J.P.; Li, X.L.; Li, G.H.; Chen, W.; Arakaki, C.; Botimer, G.D.; Baylink, D.; Zhang, L.; Wen, W.; Fu, Y.W.; et al. Efficient precise knockin with a double cut HDR donor after CRISPR/Cas9-mediated double-stranded DNA cleavage. Genome Biol. 2017, 18, 35. [CrossRef] [PubMed]

22. Vartak, S.V.; Raghavan, S.C. Inhibition of nonhomologous end joining to increase the specificity of CRISPR/Cas9 genome editing. FEBS J. 2015, 282, 4289-4294. [CrossRef] [PubMed]

23. Chu, V.T.; Weber, T.; Wefers, B.; Wurst, W.; Sander, S.; Rajewsky, K.; Kuhn, R. Increasing the efficiency of homology-directed repair for CRISPR-Cas9-induced precise gene editing in mammalian cells. Nat. Biotechnol. 2015, 33, 543-548. [CrossRef]

24. Lin, S.; Staahl, B.T.; Alla, R.K.; Doudna, J.A. Enhanced homology-directed human genome engineering by controlled timing of CRISPR/Cas9 delivery. eLife 2014, 3, e04766. [CrossRef] [PubMed]

25. Shao, S.; Ren, C.; Liu, Z.; Bai, Y.; Chen, Z.; Wei, Z.; Wang, X.; Zhang, Z.; Xu, K. Enhancing CRISPR/Cas9mediated homology-directed repair in mammalian cells by expressing Saccharomyces cerevisiae Rad52. Int. J. Biochem. Cell Biol. 2017, 92, 43-52. [CrossRef]

26. Di Primio, C.; Galli, A.; Cervelli, T.; Zoppe, M.; Rainaldi, G. Potentiation of gene targeting in human cells by expression of Saccharomyces cerevisiae Rad52. Nucleic Acids Res. 2005, 33, 4639-4648. [CrossRef]

27. Nambiar, T.S.; Billon, P.; Diedenhofen, G.; Hayward, S.B.; Taglialatela, A.; Cai, K.; Huang, J.W.; Leuzzi, G.; Cuella-Martin, R.; Palacios, A.; et al. Stimulation of CRISPR-mediated homology-directed repair by an engineered RAD18 variant. Nat. Commun. 2019, 10, 3395. [CrossRef]

28. Charpentier, M.; Khedher, A.H.Y.; Menoret, S.; Brion, A.; Lamribet, K.; Dardillac, E.; Boix, C.; Perrouault, L.; Tesson, L.; Geny, S.; et al. CtIP fusion to Cas9 enhances transgene integration by homology-dependent repair. Nat. Commun. 2018, 9, 1133. [CrossRef]

29. Pierce, A.J.; Johnson, R.D.; Thompson, L.H.; Jasin, M. XRCC3 promotes homology-directed repair of DNA damage in mammalian cells. Genes Dev. 1999, 13, 2633-2638. [CrossRef]

30. Buchholz, D.R. Tet-On binary systems for tissue-specific and inducible transgene expression. Methods Mol. Biol. 2012, 917, 265-275. [CrossRef]

31. Willis, N.A.; Chandramouly, G.; Huang, B.; Kwok, A.; Follonier, C.; Deng, C.; Scully, R. BRCA1 controls homologous recombination at Tus/Ter-stalled mammalian replication forks. Nature 2014, 510, 556-559. [CrossRef] [PubMed]

32. Zhao, W.; Steinfeld, J.B.; Liang, F.; Chen, X.; Maranon, D.G.; Jian Ma, C.; Kwon, Y.; Rao, T.; Wang, W.; Sheng, C.; et al. BRCA1-BARD1 promotes RAD51-mediated homologous DNA pairing. Nature 2017, 550, 360-365. [CrossRef] [PubMed]

33. Anand, R.; Beach, A.; Li, K.; Haber, J. Rad51-mediated double-strand break repair and mismatch correction of divergent substrates. Nature 2017, 544, 377-380. [CrossRef] [PubMed]

34. Sartori, A.A.; Lukas, C.; Coates, J.; Mistrik, M.; Fu, S.; Bartek, J.; Baer, R.; Lukas, J.; Jackson, S.P. Human CtIP promotes DNA end resection. Nature 2007, 450,509-514. [CrossRef]

35. Yun, M.H.; Hiom, K. CtIP-BRCA1 modulates the choice of DNA double-strand-break repair pathway throughout the cell cycle. Nature 2009, 459, 460-463. [CrossRef]

36. Richardson, C.D.; Kazane, K.R.; Feng, S.J.; Zelin, E.; Bray, N.L.; Schafer, A.J.; Floor, S.N.; Corn, J.E. CRISPR-Cas9 genome editing in human cells occurs via the Fanconi anemia pathway. Nat. Genet. 2018, 50, 1132-1139. [CrossRef]

37. Zhu, S.; Li, W.; Liu, J.; Chen, C.H.; Liao, Q.; Xu, P.; Xu, H.; Xiao, T.; Cao, Z.; Peng, J.; et al. Genome-scale deletion screening of human long non-coding RNAs using a paired-guide RNA CRISPR-Cas9 library. Nat. Biotechnol. 2016, 34, 1279-1286. [CrossRef]

38. Lee, C.; Le, M.P.; Wallingford, J.B. The shroom family proteins play broad roles in the morphogenesis of thickened epithelial sheets. Dev. Dyn. 2009, 238, 1480-1491. [CrossRef]

39. Lee, C.; Scherr, H.M.; Wallingford, J.B. Shroom family proteins regulate gamma-tubulin distribution and microtubule architecture during epithelial cell shape change. Development 2007, 134, 1431-1441. [CrossRef] 
40. Hildebrand, J.D.; Soriano, P. Shroom, a PDZ domain-containing actin-binding protein, is required for neural tube morphogenesis in mice. Cell 1999, 99, 485-497. [CrossRef]

41. Zalewski, J.K.; Mo, J.H.; Heber, S.; Heroux, A.; Gardner, R.G.; Hildebrand, J.D.; VanDemark, A.P. Structure of the Shroom-Rho Kinase Complex Reveals a Binding Interface with Monomeric Shroom That Regulates Cell Morphology and Stimulates Kinase Activity. J. Biol. Chem. 2016, 291, 25364-25374. [CrossRef] [PubMed]

42. Thwaites, D.T.; Anderson, C.M. The SLC36 family of proton-coupled amino acid transporters and their potential role in drug transport. Br. J. Pharmacol. 2011, 164, 1802-1816. [CrossRef] [PubMed]

43. Bermingham, J.R., Jr.; Pennington, J. Organization and expression of the SLC36 cluster of amino acid transporter genes. Mamm. Genome 2004, 15, 114-125. [CrossRef] [PubMed]

44. Stachelek, G.C.; Peterson-Roth, E.; Liu, Y.; Fernandez, R.J., 3rd; Pike, L.R.; Qian, J.M.; Abriola, L.; Hoyer, D.; Hungerford, W.; Merkel, J.; et al. YU238259 Is a Novel Inhibitor of Homology-Dependent DNA Repair That Exhibits Synthetic Lethality and Radiosensitization in Repair-Deficient Tumors. Mol. Cancer Res. 2015, 13, 1389-1397. [CrossRef] [PubMed]

45. Beucher, A.; Birraux, J.; Tchouandong, L.; Barton, O.; Shibata, A.; Conrad, S.; Goodarzi, A.A.; Krempler, A.; Jeggo, P.A.; Lobrich, M. ATM and Artemis promote homologous recombination of radiation-induced DNA double-strand breaks in G2. EMBO J. 2009, 28, 3413-3427. [CrossRef]

(C) 2020 by the authors. Licensee MDPI, Basel, Switzerland. This article is an open access article distributed under the terms and conditions of the Creative Commons Attribution (CC BY) license (http://creativecommons.org/licenses/by/4.0/). 